\title{
Reynolds and Prandtl number scaling of viscous heating in isotropic turbulence
}

\author{
Andrey Pushkarev, ${ }^{1}$ Guillaume Balarac, ${ }^{1}$ and Wouter J. T. Bos ${ }^{2}$ \\ ${ }^{1}$ LEGI, CNRS, Grenoble INP, 38000 Grenoble, France \\ ${ }^{2}$ LMFA, CNRS, Ecole Centrale de Lyon, Université de Lyon, 69134 Ecully, France
}

(Received 14 April 2017; published 21 August 2017)

\begin{abstract}
Viscous heating is investigated using high-resolution direct numerical simulations. Scaling relations are derived and verified for different values of the Reynolds and Prandtl numbers. The scaling of the heat fluctuations is shown to depend on Lagrangian correlation times and on the scaling of dissipation-rate fluctuations. The convergence of the temperature spectrum to asymptotic scaling is observed to be slow, due to the broadband character of the temperature production spectrum and the slow convergence of the dissipation-rate spectrum to its asymptotic form.
\end{abstract}

DOI: 10.1103/PhysRevFluids.2.084606

\section{INTRODUCTION}

The equation governing the heat fluctuations $\theta$ generated by an incompressible, statistically isotropic turbulent velocity field $\boldsymbol{u}$ is

$$
\partial_{t} \theta+\boldsymbol{u} \cdot \nabla \theta=D \Delta \theta+\epsilon^{\prime} / c_{p}
$$

where $c_{p}$ is the heat capacity and $D$ the thermal diffusivity. The source term is proportional to the dissipation-rate fluctuation $\epsilon^{\prime}=\epsilon-\langle\epsilon\rangle$, where the angular brackets denote an ensemble average. Recent studies investigated this system using spectral closures [1,2]. In Ref. [1] a spectral model was used, derived using dimensional arguments. In Ref. [2] one of us derived the eddy-damped quasinormal Markovian expression for the viscous heat production. Neither of these approaches was capable of taking into account the correct spatial distribution of dissipation-rate fluctuations, which turn out to be essential for a correct representation of the viscous heat generation. Whereas the influence of the spatial distribution of the dissipation-rate fluctuations on the statistics of second-order correlations such as the kinetic energy is in general small, the actual wave-number spectrum of the dissipation-rate fluctuations can differ dramatically from closure predictions [3]; this effect should carry over to the scaling of the temperature fluctuations [4].

In a previous investigation [4], numerical simulations were carried out to quantify the viscous heat production for unity Prandtl number and Reynolds numbers up to $\operatorname{Re}_{\lambda}=77$. It was clearly illustrated that the large-scale correlation of the dissipation-rate fluctuations should be taken into account to properly model the viscous heat production. What was not clear was how to choose the Lagrangian correlation time of the heat fluctuations. Furthermore, the relatively low Reynolds numbers did not allow determination of the exact scaling of the variance and dissipation of the heat fluctuations.

In this paper we present results from direct numerical simulations at considerably higher Reynolds numbers $\left(\operatorname{Re}_{\lambda} \leqslant 370\right)$, supplemented by a variety of different Prandtl numbers in the range $0.001 \leqslant$ $\operatorname{Pr} \leqslant 12$. This allows us to verify the predictions of the precedent investigation, for instance, the role and behavior of the Lagrangian correlation time associated with the heat production, and will allow us to assess the influence of the Prandtl number on the heat production.

We will first, in the following section, discuss the scaling of dissipation-rate fluctuations. Then, in Sec. III, we derive the expected scaling of the variance and dissipation of temperature fluctuations, in the light of the insights obtained in Ref. [4]. In Sec. IV the numerical method and parameters are discussed and in Sec. V the results of the simulations are reported. Section VI summarizes the paper. 


\section{SCALING OF THE DISSIPATION-RATE FLUCTUATIONS}

A correct representation of the spatial correlation of the dissipation-rate fluctuations will be essential in our description of frictional heating. We therefore consider the spectrum of the dissipation-rate fluctuations, defined such that

$$
\int E_{\epsilon}(k) d k=\left\langle\epsilon^{\prime 2}\right\rangle
$$

This quantity can be expressed as a function of fourth-order velocity-gradient correlations. Assuming the velocity statistics to be joint Gaussian, the dissipation-rate spectrum (2) can be expressed as a function of the kinetic energy spectrum. Assuming Kolmogorov scaling for the kinetic energy spectrum, one obtains the inertial range scaling [3]

$$
E_{\epsilon}(k) \sim v^{2} \varepsilon^{4 / 3} k^{5 / 3} .
$$

Since the mean dissipation will appear in a large number of expressions in the following, we have introduced the shorthand notation $\varepsilon=\langle\epsilon\rangle$. In the following, we will express quantities, where possible, as a function of the Kolmogorov length scale $\eta=v^{3 / 4} \varepsilon^{-1 / 4}$ and the mean dissipation $\varepsilon$. For the Gaussian estimate of $E_{\epsilon}(k)$ [Eq. (3)] we obtain then the expression

$$
E_{\epsilon}(k) \sim \varepsilon^{2} \eta(k \eta)^{5 / 3} \text {. }
$$

The fact that this is an increasing function of $k$ suggests that the dissipation-rate fluctuations are dominated by spatial correlations at small scales, around $k_{\eta}$, the Kolmogorov scale. In reality, it is observed in experiments and simulations [3,5-8] that the dissipation-rate fluctuations are correlated at large scales, following a power law better described by

$$
E_{\epsilon}(k) \sim \varepsilon^{2} L(k L)^{-1+\mu},
$$

where the coefficient $0 \leqslant \mu<1$ is either determined experimentally, or derived from empirical models. In this expression $L$ is the integral length scale

$$
L=\frac{3 \pi}{4} \frac{\int k^{-1} E(k) d k}{\int E(k) d k},
$$

where $E(k)$ is the kinetic energy spectrum. No derivation of the value of $\mu$ from the Navier-Stokes equations exists. A commonly used model to determine the quantitative influence of intermittency is due to She and Lévêque [9], yielding the value $\mu=2 / 9$. We see that, using this value, the dissipation-rate spectrum, instead of being an increasing function of $k$ proportional to $k^{5 / 3}$, is a decreasing function, roughly proportional to $k^{-7 / 9}$.

This enormous difference between the Gaussian estimate (3) and expression (5), irrespective of the precise value of $\mu$, leads to a completely erroneous prediction of the heat generated by viscous friction. ${ }^{1}$ It was shown that for this reason spectral models underestimate, for high-Reynolds-number flows, the heat production by several orders of magnitude [4]. Technically, the reason is that this type of spectral closure is not able to take into account all cumulant corrections to fourth-order velocity correlations $[10,11]$.

In Ref. [4] we used the expression (5), which was, we thought, a precise enough description of the dissipation-rate spectrum. According to the expression (5), the dissipation-rate fluctuations are entirely determined by the quantities $\varepsilon$ and $L$. In high-resolution direct numerical simulations (DNSs), it was however shown that this might not be the case [12]. Indeed, in those simulations of

\footnotetext{
${ }^{1}$ The parameter $\mu$ is generally called the intermittency coefficient, so one could call the case $\mu=0$ the nonintermittent case. However, even for $\mu=0$ the spectrum (3) is completely different from (5). We will not try to answer the question whether this difference between the Gaussian estimate and the actual spectrum is due to internal intermittency or to another effect.
} 
isotropic turbulence with Reynolds numbers up to $\operatorname{Re}_{\lambda}=732$, it was shown that the dissipation-rate spectrum scales as

$$
E_{\epsilon}(k) \sim \varepsilon^{2} \eta(k \eta)^{-1+\mu} \operatorname{Re}_{\lambda}^{1 / 4}
$$

where $\mu=1 / 3$ and the Reynolds number is given by

$$
\operatorname{Re}_{\lambda}=\sqrt{15} \frac{U^{2}}{\sqrt{\varepsilon v}},
$$

with $U$ the root-mean-square value of a velocity component. The expression (7) differs from (5) by a Reynolds-number correction. Indeed, expressing (7) as a function of $L$ instead of $\eta$, using that $L \sim \operatorname{Re}_{\lambda}^{3 / 2} \eta$, it is found that

$$
E_{\epsilon}(k) \sim \varepsilon^{2} L(k L)^{-2 / 3} \operatorname{Re}_{\lambda}^{-1 / 4},
$$

which corresponds to (5), with $\mu=1 / 3$, multiplied by $\operatorname{Re}_{\lambda}^{-1 / 4}$. This will alter our estimations.

\section{ANALYSIS OF THE SPECTRAL TEMPERATURE BUDGET}

\section{A. Temperature balance equation}

The present investigation focuses on wave-number spectra of fluctuating quantities. In order to better understand the scaling of these spectra, we first write Eq. (1) in Fourier space

$$
\partial_{t} \theta(\boldsymbol{k})=-i k_{i} \int u_{i}(\boldsymbol{p}) \theta(\boldsymbol{k}-\boldsymbol{p}) d \boldsymbol{p}-D k^{2} \theta(\boldsymbol{k})+\epsilon^{\prime}(\boldsymbol{k}) / c_{p},
$$

where the Fourier transformed quantities can be recognized by their dependence on the wave vector. The spectra of the kinetic energy and temperature variance are defined such that

$$
\int E(k) d k=\frac{1}{2}\left\langle u_{i} u_{i}\right\rangle, \quad \int E_{\theta}(k) d k=\frac{1}{2}\left\langle\theta^{2}\right\rangle
$$

The equation for the time evolution of the temperature spectrum is then

$$
\partial_{t} E_{\theta}(k)=T_{\theta}(k)-2 D k^{2} E_{\theta}(k)+P_{\theta}(k),
$$

where

$$
T_{\theta}(k)=-4 i \pi k^{2} k_{i} \int\left\langle\theta(-\boldsymbol{k}) u_{i}(\boldsymbol{p}) \theta(\boldsymbol{k}-\boldsymbol{p})\right\rangle d \boldsymbol{p}
$$

and

$$
P_{\theta}(k)=4 \pi k^{2}\left\langle\epsilon^{\prime}(\boldsymbol{k}) \theta(-\boldsymbol{k})\right\rangle / c_{p} .
$$

The wave-number integral of expression (12) gives the equation for the scalar variance

$$
d_{t}\left\langle\theta^{2}\right\rangle=p_{\theta}-\varepsilon_{\theta},
$$

where $p_{\theta}=\int P_{\theta}(k) d k$ and $\varepsilon_{\theta}=\int 2 D k^{2} E_{\theta}(k) d k$. In a steady state we naturally have a balance $p_{\theta}=\varepsilon_{\theta}$. In the following we will evaluate the production spectrum $P_{\theta}(k)$. Since we consider only statistically stationary states, its integral will directly determine the scalar dissipation.

\section{B. Model for production}

Following [4], we write the contribution of $\theta(\boldsymbol{k}, t)$ as

$$
\theta(\boldsymbol{k}, t)=\theta(\boldsymbol{k}, t \mid 0)+c_{p}^{-1} \int_{0}^{t} G_{\theta}(\boldsymbol{k}, t \mid s) \epsilon^{\prime}(\boldsymbol{k}, t \mid s) d s,
$$


where $G_{\theta}(\boldsymbol{k}, t \mid s)$ is the scalar Green's function and $f(\boldsymbol{k}, t \mid s)$ is the Fourier transform with respect to $\boldsymbol{x}$ of a quantity $f(\boldsymbol{x}, t)$ evaluated at time $s$ on the trajectory, passing at a time $t$ through position $\boldsymbol{x}$. We find then for the production term

$$
P_{\theta}(k)=\frac{1}{c_{p}^{2}} \Theta(k) E_{\epsilon}(k)
$$

where

$$
\Theta(k)=\int_{0}^{t} G_{\theta}(k, t \mid s) R_{\epsilon}(k, t \mid s) d s
$$

with

$$
R_{\epsilon}(k, t \mid s)=\frac{E_{\epsilon}(k, t \mid s)}{E_{\epsilon}(k, t)} .
$$

Assuming exponential time correlations for $R_{\epsilon}(k, t \mid s)$ and $G(k, t \mid s)$, both proportional to $\sim \exp (-\mid t-$ $s \mid / \tau)$, we find

$$
\Theta(k)=\frac{1}{\tau_{\theta}(k)^{-1}+\tau_{\epsilon}(k)^{-1}} .
$$

The precise functional form (exponential, Gaussian, or alike) is not very important in the derivation and will lead to similar expressions as long as the correlation functions decrease sufficiently rapidly for $t / \tau \gg 1$. Up to this point no important simplifications are introduced. However, the transfer is undetermined and so are the time scales $\tau_{\theta}$ and $\tau_{\epsilon}$. The dissipation fluctuation spectrum $E_{\epsilon}(k)$ was discussed in the preceding section.

We start with the important question of how to define the time scales. In Ref. [4] it was assumed that the correlation time of the dissipation-rate fluctuations $\tau_{\epsilon}$ was of the order of the integral time scale. Even if this is so, there is a second time scale $\tau_{\theta}$, related to the scalar decorrelation appearing in the expression (20), which was not taken into account in the previous investigation. The dominant time scale is determined by the sum of their inverse values [see Eq. (20)]. It is plausible to assume that the correlation time $\tau_{\theta}$, related to the time it takes for a temperature fluctuation to decorrelate from its trajectory, is influenced by eddy diffusion, i.e., the diffusive influence of velocity fluctuations encountered on its trajectory. By decorrelating we mean the loss of correlation, in time, in a statistical sense with respect to the point $\boldsymbol{x}$ at point $t$, following the fluid particle back on its trajectory [13]. If eddy diffusion is taken into account, the scalar time scale should be, in the inertial range, proportional to the typical Lagrangian straining time

$$
\tau_{\theta}(k) \sim \varepsilon^{-1 / 3} k^{-2 / 3} .
$$

Such a time becomes for large $k$ dominant over the integral time in the expression (20), which depends on the sum of the inverse values of the time scales, and this will change the analysis with respect to Ref. [4].

\section{Temperature spectrum in the inertial-convective range (unity and large values of the Prandtl number)}

Let us consider the ideal case of a long inertial-convective range where only production and transfer are important, ignoring the influence of the shape of the spectra for small wave numbers. We write the scalar transfer as the derivative of a flux

$$
T_{\theta}(k)=-\partial_{k} \Pi_{\theta}(k) .
$$

A balance between transfer and production $T_{\theta}(k)=-P_{\theta}(k)$ then yields

$$
\Pi_{\theta}(k)=\int_{k_{0}}^{k} P_{\theta}(k) d k,
$$


where $k_{0}$ marks the beginning of the inertial range. Assuming that all quantities behave as power laws in the inertial range, the flux will tend to a constant value, independent of the wave number $k$ if $P_{\theta}(k) \sim k^{\alpha}$ with $\alpha<-1$. In that case $\Pi_{\theta}(k)=\varepsilon_{\theta}=p_{\theta}$. For values of $\alpha \geqslant-1$, the flux will be an increasing function of $k$. In Ref. [4] this seemed to be the case. We will not confirm that observation here.

Combining Eq. (17) for the production with the expression (9) for $E_{\epsilon}(k)$, we obtain

$$
\Pi_{\theta}(k) \sim \frac{\varepsilon^{2} \eta}{c_{p}^{2}} \operatorname{Re}_{\lambda}^{1 / 4} \int_{k_{0}}^{k} \Theta(k)(k \eta)^{\mu-1} d k .
$$

Assuming that the Lagrangian time scale in the inertial range will be dominated by the spectrally local time scale (21), the value of the flux tends to a constant value in the inertial range

$$
\Pi_{\theta}(k) \sim \frac{\varepsilon^{5 / 3} \eta^{2 / 3}}{c_{p}^{2}} \operatorname{Re}_{\lambda}^{(5-6 \mu) / 4}
$$

for $k \gg k_{0}$ and $\mu<2 / 3$ and using that $1 / k_{0} \sim L \sim \operatorname{Re}_{\lambda}^{3 / 2} \eta$. We have therefore

$$
p_{\theta}=\varepsilon_{\theta} \approx \Pi_{\theta}(k) \sim \frac{\varepsilon^{5 / 3} \eta^{2 / 3}}{c_{p}^{2}} \operatorname{Re}_{\lambda}^{(5-6 \mu) / 4} .
$$

A constant scalar flux is the prerequisite for Corrsin-Obukhov scaling [14,15],

$$
E_{\theta}(k) \sim \varepsilon_{\theta} \varepsilon^{-1 / 3} k^{-5 / 3},
$$

where $\varepsilon_{\theta}$ is given by (26). Combining the last two expressions and integrating, we find that the scalar variance is then given by

$$
\begin{aligned}
\left\langle\theta^{2}\right\rangle & \sim \varepsilon_{\theta} \varepsilon^{-1 / 3} \eta^{2 / 3} \operatorname{Re}_{\lambda} \\
& \sim \frac{(\varepsilon \eta)^{4 / 3}}{c_{p}^{2}} \operatorname{Re}_{\lambda}^{(9-6 \mu) / 4}
\end{aligned}
$$

\section{Temperature spectrum in the Batchelor range}

In the case where the Prandtl number is very large, a correction to this expression can appear. Indeed, for $\operatorname{Pr} \gg 1$, a second scaling range is expected to appear, proportional to

$$
E_{\theta}(k) \sim \varepsilon_{\theta} \sqrt{v / \varepsilon} k^{-1}
$$

for $k \gg k_{\eta}$, up to the Batchelor scale $k_{B} \approx \operatorname{Pr}^{1 / 2} k_{\eta}$. The scalar variance contained in this range can be estimated by

$$
\begin{aligned}
\left\langle\theta^{2}\right\rangle^{B} & \sim \varepsilon_{\theta} \sqrt{\nu / \varepsilon} \int_{k_{\eta}}^{k_{B}} k^{-1} d k \\
& \sim \varepsilon_{\theta} \sqrt{\nu / \varepsilon} \ln \left(\operatorname{Pr}^{1 / 2}\right) .
\end{aligned}
$$

Comparing the contributions in the inertial convective and Batchelor range, we find that

$$
\frac{\left\langle\theta^{2}\right\rangle^{B}}{\left\langle\theta^{2}\right\rangle} \sim \operatorname{Re}_{\lambda}^{-1} \ln \left(\operatorname{Pr}^{1 / 2}\right)
$$

This shows that the variance contained in the Batchelor range can be safely ignored for the case we consider, where $\operatorname{Pr} \leqslant 12$ and $\operatorname{Re}_{\lambda} \gg 1$. We will therefore, if our estimations are precise enough, not find any significant contribution to the scalar variance related to the Batchelor range. 


\section{E. Viscous heating at very small Prandtl numbers}

In the case of a very diffusive temperature, i.e., $D \gg v$, a balance is expected between the diffusion of temperature and the viscous heat production. This means that in Eq. (10) the last two terms dominate over the other terms (cf. the ideas in Ref. [16]),

$$
D k^{2} \theta(\boldsymbol{k}, t) \approx \epsilon^{\prime}(\boldsymbol{k}, t) / c_{p} .
$$

Squaring both sides, it is immediately found that

$$
2 D^{2} k^{4} E_{\theta}(k, t) \approx E_{\epsilon}(k, t) / c_{p}^{2},
$$

so that, combined with the expression (7), we find

$$
E_{\theta}(k \eta) \sim \frac{\varepsilon^{4 / 3} \eta^{7 / 3}}{c_{p}^{2}}(k \eta)^{\mu-5} \operatorname{Pr}^{2} \operatorname{Re}_{\lambda}^{1 / 4} .
$$

Integrating this from $k_{0}$ to $k_{\eta}$ gives

$$
\left\langle\theta^{2}\right\rangle \sim \frac{(\varepsilon \eta)^{4 / 3}}{c_{p}^{2}} \operatorname{Re}_{\lambda}^{(25-6 \mu) / 4} \operatorname{Pr}^{2}
$$

and for the dissipation

$$
\varepsilon_{\theta} \sim \frac{\varepsilon^{5 / 3} \eta^{2 / 3}}{c_{p}^{2}} \operatorname{Re}_{\lambda}^{(13-6 \mu) / 4} \operatorname{Pr} .
$$

\section{F. Predictions for the scaling of integral quantities}

In the following, the relations that we will check in our simulations concern the variance and dissipation of temperature fluctuations. We have chosen to express all variables in Kolmogorov variables $\varepsilon$ and $\eta$. All expressions can be recast in terms of $U$ and $L$, using that

$$
\varepsilon \sim \frac{U^{3}}{L}
$$

a relation that will be shown to hold in our simulations (see Fig. 1). Since this further implies

$$
\operatorname{Re}_{\lambda}^{2} \operatorname{Pr} \sim \frac{U L}{D} \equiv \operatorname{Pe},
$$

where Pe is the Péclet number, we can write the expressions to be verified as

$$
\frac{\left\langle\theta^{2}\right\rangle}{(\varepsilon \eta)^{4 / 3} c_{p}^{-2}} \sim \begin{cases}\operatorname{Re}_{\lambda}^{7 / 4} & \text { for } \operatorname{Pr} \approx 1 \\ \operatorname{Re}_{\lambda}^{7 / 4}\left[1+O\left(\operatorname{Re}_{\lambda}^{-1} \ln \left(\operatorname{Pr}^{1 / 2}\right)\right)\right] & \text { for } \operatorname{Pr} \gg 1 \\ \operatorname{Re}_{\lambda}^{7 / 4} \operatorname{Pe}^{2} & \text { for } \operatorname{Pr} \ll 1\end{cases}
$$

and for the scalar dissipation

$$
\frac{\varepsilon_{\theta}}{\varepsilon^{5 / 3} \eta^{2 / 3} c_{p}^{-2}} \sim\left\{\begin{array}{lc}
\operatorname{Re}_{\lambda}^{3 / 4} & \text { for } \operatorname{Pr} \geqslant 1 \\
\operatorname{Re}_{\lambda}^{3 / 4} \mathrm{Pe} & \text { for } \operatorname{Pr} \ll 1 .
\end{array}\right.
$$

In these expressions we have used the value $\mu=1 / 3$, observed in the simulations in Ref. [12].

\section{NUMERICAL METHOD AND PARAMETERS}

We assess the proposed scaling relations for the variance and the dissipation of temperature fluctuations using a DNS database of forced homogeneous isotropic turbulence. We carried out standard pseudospectral simulations in a cubic domain of size $2 \pi$. A statistical steady flow is 


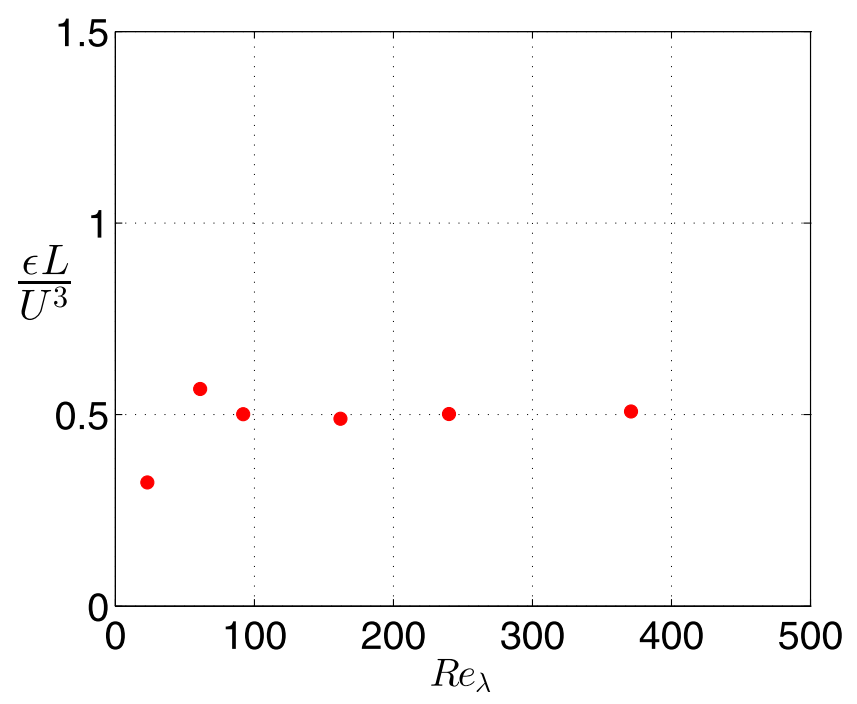

(a)

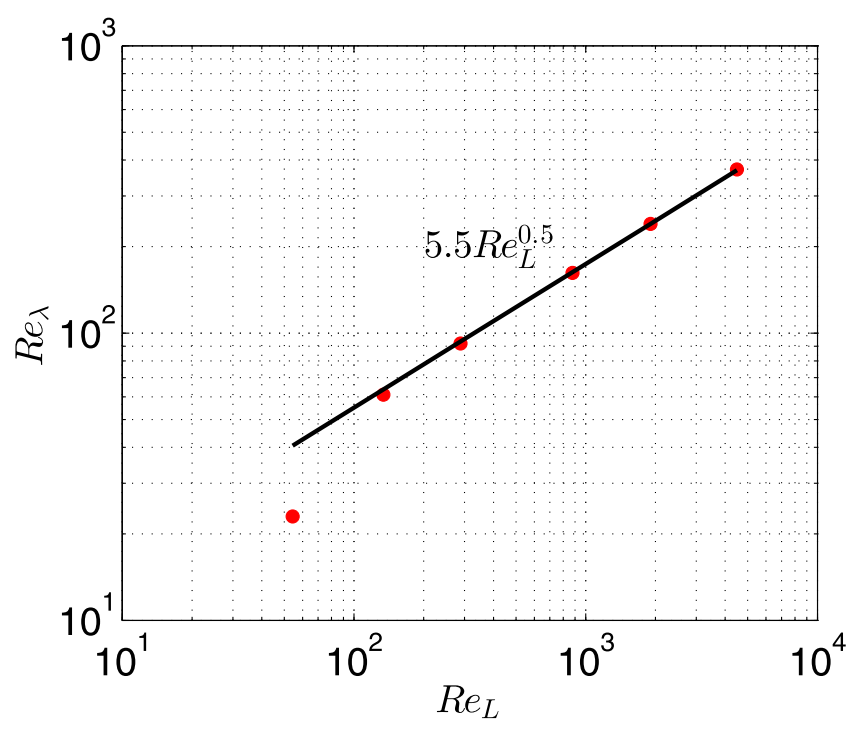

(b)

FIG. 1. (a) Normalized dissipation rate as a function of the Taylor-scale Reynolds number. (b) Taylor-scale Reynolds number as a function of the integral Reynolds number.

achieved by using a random forcing scheme applied at low wave numbers, around $k=k_{f}$ [17]. The temperature, modeled as a passive scalar, is initialized at zero value. Its variance grows until a statistically steady state is attained, where on average the temperature source term $p_{\theta}$ associated with the last term in Eq. (1) is balanced by the dissipation of temperature fluctuations $\varepsilon_{\theta}$. The statistics are computed during this steady state.

Reynolds numbers ranging from $\operatorname{Re}_{\lambda}=30$ to 370 are considered, using a spatial resolution varying between $64^{3}$ and $2048^{3}$ grid points. Prandtl numbers are considered between $\operatorname{Pr}=0.001$ 
TABLE I. Parameters of the simulations.

\begin{tabular}{lccc}
\hline \hline $\operatorname{Re}_{\lambda}$ & $\operatorname{Pr}$ & $\begin{array}{c}N_{x y z} \\
\text { (scalar field) }\end{array}$ & $\begin{array}{c}N_{x y z} \\
\text { (velocity field) }\end{array}$ \\
\hline 30 & $0.001,0.01,0.1,1$ & $64^{3}$ & $64^{3}$ \\
& $2,4,12$ & $256^{3}$ & \\
60 & $0.001,0.01,0.1,1$ & $128^{3}$ & $128^{3}$ \\
& $2,4,12$ & $512^{3}$ & \\
90 & $1,2,4,12$ & $1024^{3}$ & $256^{3}$ \\
160 & $1,2,4,12$ & $2048^{3}$ & $512^{3}$ \\
230 & 1 & $1024^{3}$ & $1024^{3}$ \\
370 & 1 & $2048^{3}$ & $2048^{3}$ \\
\hline \hline
\end{tabular}

and 12. For Prandtl numbers larger than one, the Batchelor scale $\eta_{B}=\eta_{K} / \sqrt{\operatorname{Pr}}$ is smaller than the Kolmogorov scale $\eta_{K}$. For these cases the temperature fluctuations contain scales smaller than the smallest velocity scales and their resolution requires a finer grid than the resolution of the smallest velocity scales. For Prandtl number larger than one, we therefore use a hybrid scheme, combining a spectral method for the Navier-Stokes equations and a semi-Lagrangian particle method for the evolution of the temperature fluctuations. This method takes advantage of the Lagrangian nature of particle methods to enable a good resolution of the temperature fluctuation field at affordable numerical cost. The code and the numerical methods are detailed by Lagaert et al. [18]. Table I sums up the DNS database used in this work.

\section{RESULTS}

\section{A. Statistics of the velocity field}

We characterize the turbulent velocity field. The present simulations of a turbulent velocity field using a pseudospectral method are relatively standard. We will assess our results by comparison with results from the literature on similar flows. In Fig. 1(a) we show the relation of the normalized dissipation rate $C_{\epsilon}=L \varepsilon / U^{3}$ as a function of the Reynolds number. It is observed that we are in the high-Reynolds-number regime, where this quantity tends to a constant value around 0.5 , comparable to the results presented in Ref. [19]. The relation between $\operatorname{Re}_{\lambda}$ and $\operatorname{Re}_{L}$ is shown in Fig 1(b). We find $\operatorname{Re}_{\lambda} \approx 5.5 \operatorname{Re}_{L}^{1 / 2}$, similar to the results in Ref. [20]. Indeed, the prefactor in this expression is

$$
\frac{\operatorname{Re}_{\lambda}}{\operatorname{Re}_{L}^{1 / 2}}=\sqrt{\frac{15}{C_{\epsilon}}},
$$

which gives for $C_{\epsilon}=0.5$ the value $\approx 5.5$.

The energy spectra, normalized by Kolmogorov variables, are shown in Fig. 2(a). A collapse of the spectra over approximately all scales is observed and a power-law scaling with an exponent close to $-5 / 3$ is observed.

The spectra of the dissipation-rate fluctuations are shown in Fig. 2(b). The spectra are normalized using Kolmogorov variables, plus a Reynolds number correction, the expression (9), as proposed in Ref. [12]. The spectra collapse for the highest Reynolds numbers, but it seems that the convergence to a high-Reynolds-number asymptotic shape is much slower than for the energy spectrum, for which the different energy spectra collapsed already for the lowest Reynolds number that was considered in the present investigation. Furthermore, the power-law dependence, proportional to $k^{-2 / 3}$, has not yet been attained at these Reynolds numbers. 


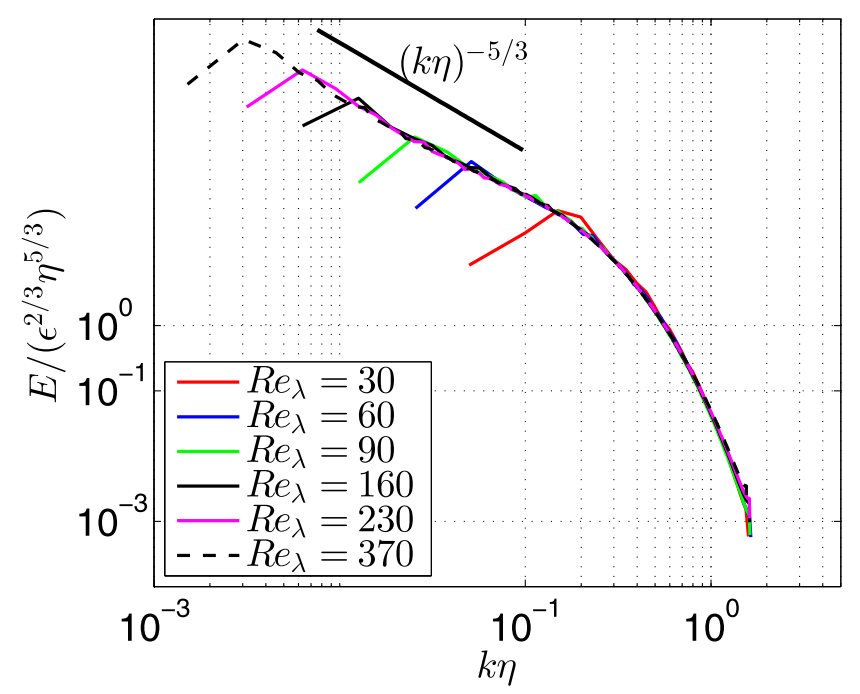

(a)

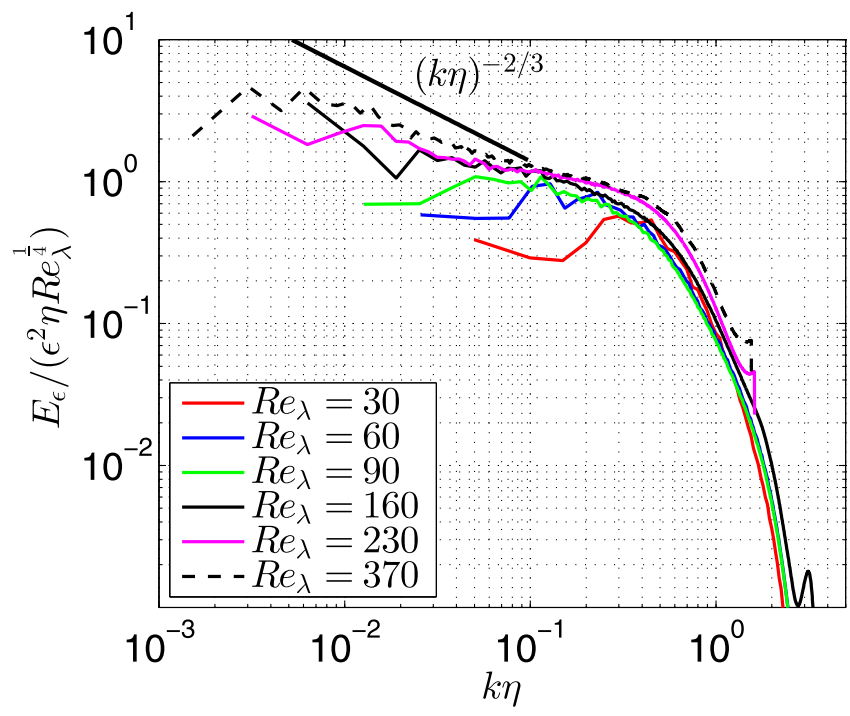

(b)

FIG. 2. (a) Energy spectra in Kolmogorov units for the different Reynolds numbers. (b) Dissipation rate fluctuation spectra, normalized according to the relation (7).

Altogether, these results are in agreement with previous computations, e.g., $[12,19,20]$. The question is now how the temperature fluctuations generated by this flow scale as a function of the different flow parameters.

\section{B. Characterization of the heat-production spectrum and the Lagrangian time scale}

In Fig. 3(a) we show the temperature production spectra $P_{\theta}(k)$ for different Reynolds numbers and $\operatorname{Pr}=1$. No clear inertial range is visible. If a power law $k^{-n}$ is to be fitted to the wave-number range where the energy spectrum shows an inertial range $(0.005 \leqslant k \eta \leqslant 0.1)$, the value of the exponent 


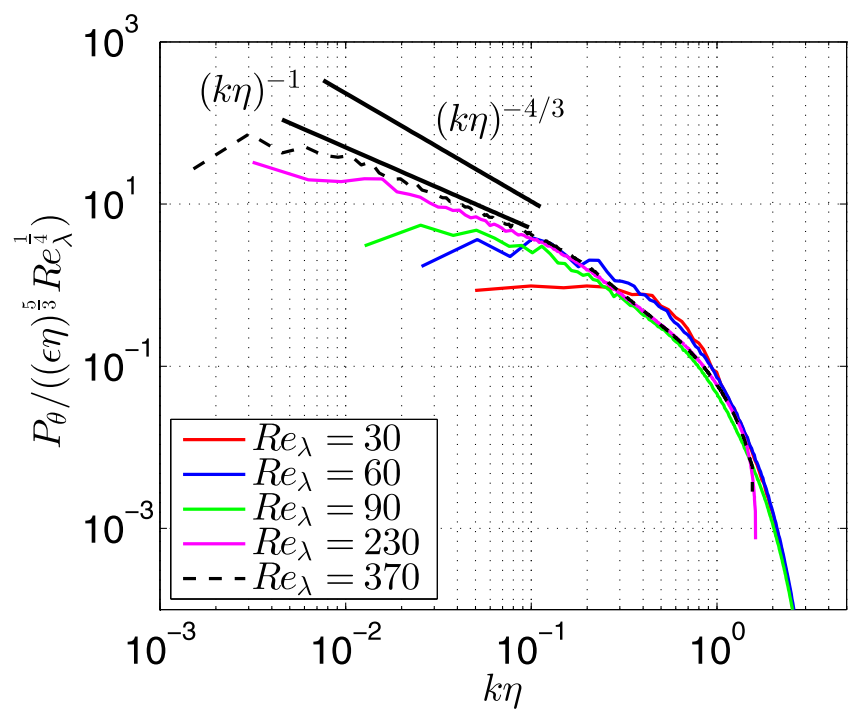

(a)

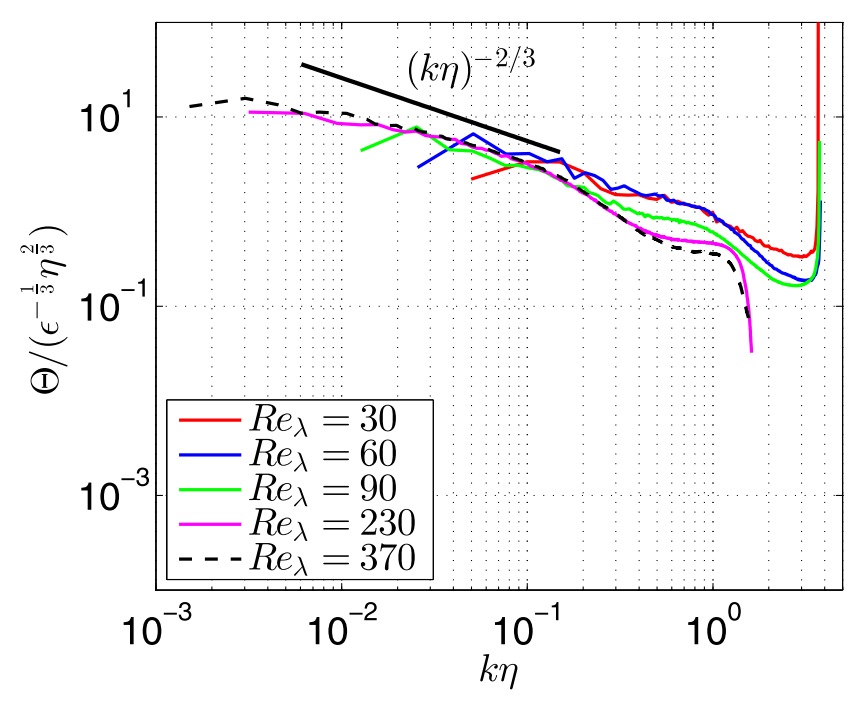

(b)

FIG. 3. (a) Temperature production spectra. (b) Lagrangian time scale, as estimated from the production spectrum and the dissipation-rate fluctuation spectrum. The results are for $\operatorname{Pr}=1$.

$n$ is closer to $n=1$ than to the exponent $n=4 / 3$, predicted by the analysis in Sec. IIIC. Both power laws are indicated in the figure. The asymptotic value, if our analysis is correct, is clearly not attained. However, the collapse of the spectra for the highest values of $\operatorname{Re}_{\lambda}$ for the range $k \eta>0.1$ consolidates the arguments in our derivation.

In this derivation we argued that the precise form of the Lagrangian correlation time $[\Theta(k)$ in the expression (17)] will determine the scaling of the temperature fluctuations. This time scale should, in the high-Reynolds-number limit, in the inertial range, be proportional to $P_{\theta}(k) / E_{\epsilon}(k)$. In Fig. 3(b) 
we plot therefore the ratio

$$
\frac{c_{p}^{2} P_{\theta}(k)}{E_{\epsilon}(k)} \sim \Theta(k),
$$

which should give an estimate of the time scale. It is shown that the time scale seems compatible with an inertial-range scaling proportional to $k^{-2 / 3}$.

This is therefore a result that invalidates the conjecture in Ref. [4] that it is the integral time scale that determines the dynamics. It is clearly a spectrally local time scale $\tau(k) \sim \varepsilon^{-1 / 3} k^{-2 / 3}$ that better fits the data.

\section{Spectrum, dissipation, and variance of temperature fluctuations}

The temperature spectra should, according to our assumptions, obey Kolmogorov-Obukhov scaling

$$
E_{\theta}(k \eta) \sim \varepsilon_{\theta} \varepsilon^{-1 / 3} \eta^{5 / 3}(k \eta)^{-n_{\theta}}
$$

This is checked in Fig. 4, where it is shown that the collapse of the spectra for large values of $k \eta$ is good for the highest values of the Reynolds number and $\operatorname{Pr}=1$. The spectra are thus determined, as far as the collapse of the data is concerned, by the quantities $\varepsilon_{\theta}, \varepsilon$, and $\nu$. For the range of scales $k \eta \ll 1$, this expression should become independent of $v$, corresponding to the Corrsin-Obukhov value $n_{\theta}=5 / 3$. This is not observed in our simulations. A better fit is obtained for $n_{\theta}=1$. We believe that this is a low-Reynolds-number effect. Indeed, the value $n_{\theta}=1$ should be observed in the Batchelor range $\operatorname{Pr} \gg 1$ for $k \eta>1$. However, in the range $k \eta$ where we observe this scaling, $k \eta<1$, the influence of the viscosity should asymptotically vanish and the value $5 / 3$ is expected to emerge. It seems from our results that the Reynolds number must be very large for this to be observed.

The very slow appearance of asymptotic scaling can be related to the way the temperature fluctuations are generated. First of all, the dissipation-rate spectrum has not obtained its asymptotic scaling [Fig. 2(b)]. We are not aware of a theoretical explanation for this slow convergence. Furthermore, even if asymptotic scaling would be obtained for $E_{\epsilon}(k)$, the temperature production spectrum is broadband and such a forcing is known to delay the emergence of asymptotic scaling regimes.

Indeed, results from high-resolution direct numerical simulations of statistically stationary isotropic turbulence mixing a passive scalar show that the temperature forcing mechanism influences the rate at which asymptotic scaling is approached [18,21]. Comparing in this context the forcing of the scalar by a large-scale spectrally local injection to the injection by means of a uniform scalar gradient, the former case, where the injection is confined to the low wave numbers, more rapidly shows convergence to Corrsin-Obukhov scaling than the second case, where the temperature forcing spectrum is broadband and approximately proportional to $k^{-7 / 3}$. This can be understood as follows: In the case of a spectrally local forcing, the range of scales where both production and dissipation can be neglected, i.e., the inertial or inertial-convective range, is larger than in the case of a broadband forcing. In the present case, the forcing is even more broadband than the mean-gradient case, since it is expected, at high Reynolds numbers, to be proportional to $k^{-4 / 3}$ (as compared to $k^{-7 / 3}$ ). The emergence of asymptotic scaling is, for this case, therefore expected to be even slower. Note that in the present case only the temperature field contains a (natural) broadband forcing term. The velocity forcing is a narrow-band energy injection and asymptotic scaling for the energy spectrum is indeed obtained to a reasonable approximation [Fig. 2(a)].

In Fig. 5(a) we show the dissipation of temperature fluctuations. It is shown that the normalization we employ reasonably well captures the Reynolds-number dependence of this quantity.

From the expression (44) we derive that

$$
\left\langle\theta^{2}\right\rangle \sim \frac{(\varepsilon \eta)^{4 / 3}}{c_{p}^{2}} \operatorname{Re}_{\lambda}^{(3 / 2)\left(n_{\theta}-1 / 2\right)}
$$




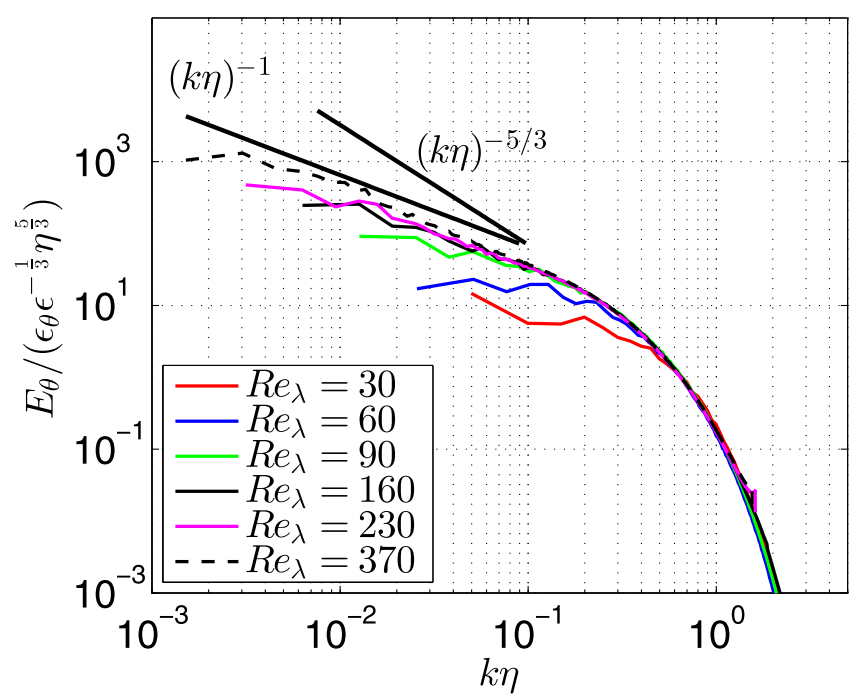

(a)

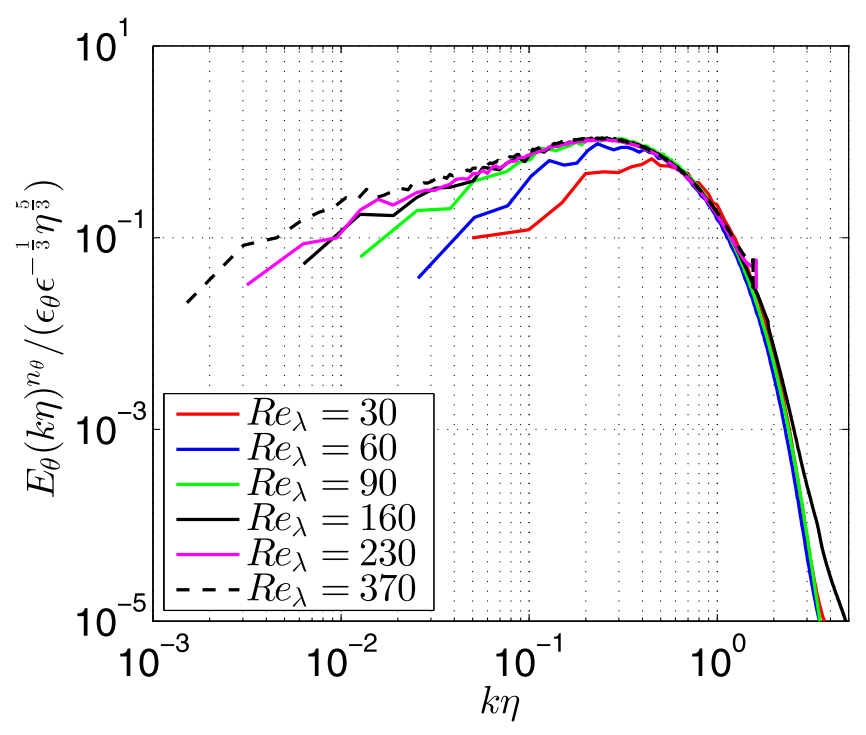

(b)

FIG. 4. Temperature fluctuation spectra in (a) Kolmogorov units and (b) compensated form. The results are for $\operatorname{Pr}=1$.

for $n_{\theta}>1$, where we have used that $L \sim \operatorname{Re}_{\lambda}^{3 / 2} \eta$. To obtain this expression we have thus assumed that our spectra scale with Corrsin-Obukhov variables, but that the Reynolds number influences the power-law exponent $n_{\theta}$. In Fig. 5(b) we show the normalized temperature variance following the expression (45). For $n=5 / 3$ the variance does not converge to a plateau. Changing the exponent to $n=4 / 3$ or $n=1$ improves the convergence of the temperature dissipation towards an asymptotic plateau, but the value 1 is completely arbitrary. The only thing this illustrates is that compensating for the Reynolds-number dependence of the power-law exponent allows us to attain asymptotic scaling for the variance of the temperature fluctuations. 


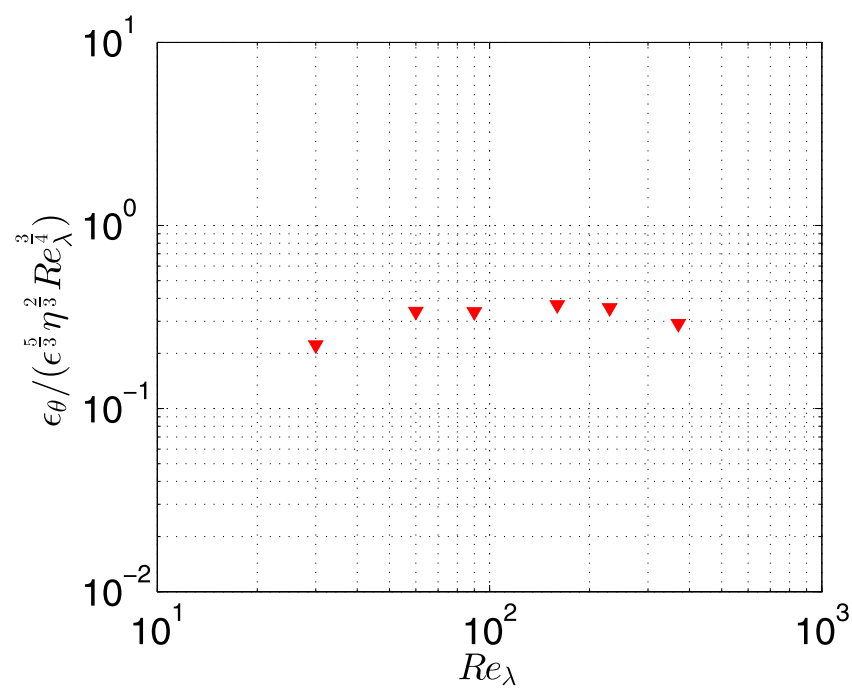

(a)

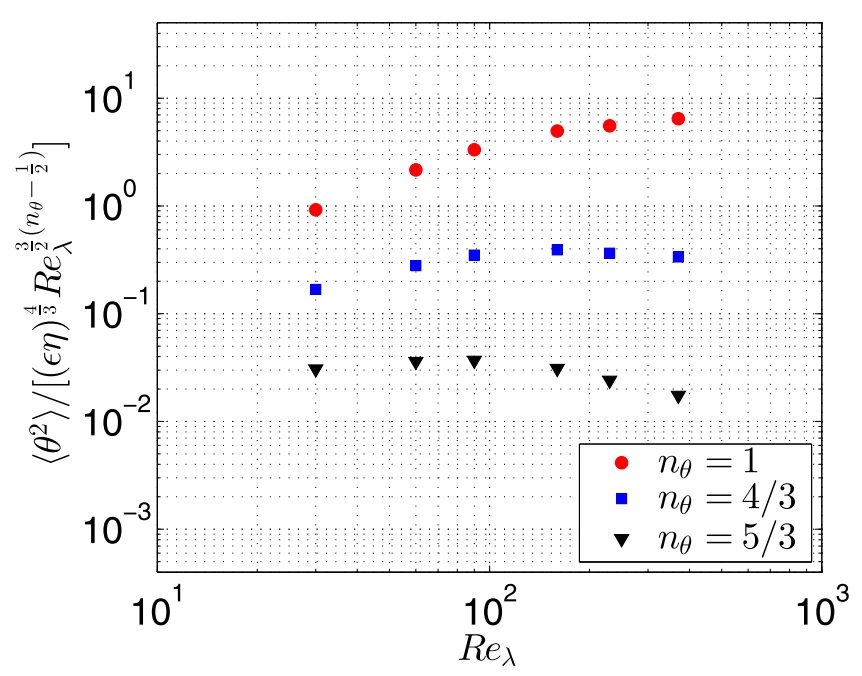

(b)

FIG. 5. (a) Reynolds-number dependence of the normalized dissipation of temperature fluctuations for $\operatorname{Pr}=1$. (b) Variance of the temperature fluctuations, normalized assuming asymptotic scaling $n_{\theta}=5 / 3$ or taking into account low-Reynolds-number corrections on the power-law exponent.

\section{Prandtl-number effects}

The analysis in Sec. III E suggested that the temperature dissipation rate should scale as a function of the Reynolds number and the Péclet number. We therefore test, in Fig. 6(a), the functional relation

$$
\varepsilon_{\theta} \frac{c_{p}^{2}}{\varepsilon^{5 / 3} \eta^{2 / 3}} \operatorname{Re}_{\lambda}^{-3 / 4}=F(\mathrm{Pe}) .
$$

This representation should, if the scaling arguments are correct, collapse the data on two asymptotic regimes: For small values of Pe, a linear dependence on Pe should be observed, whereas for large 


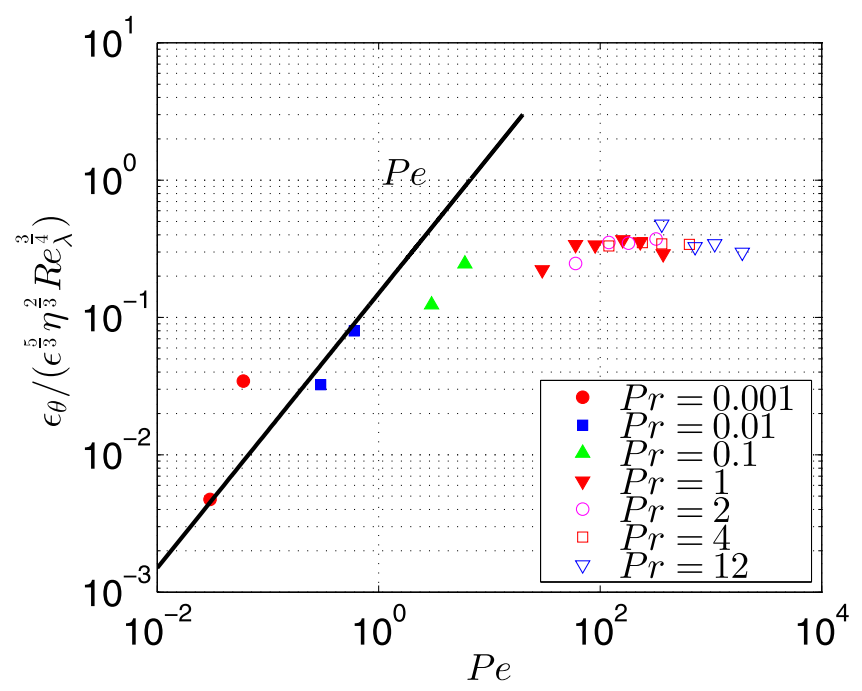

(a)

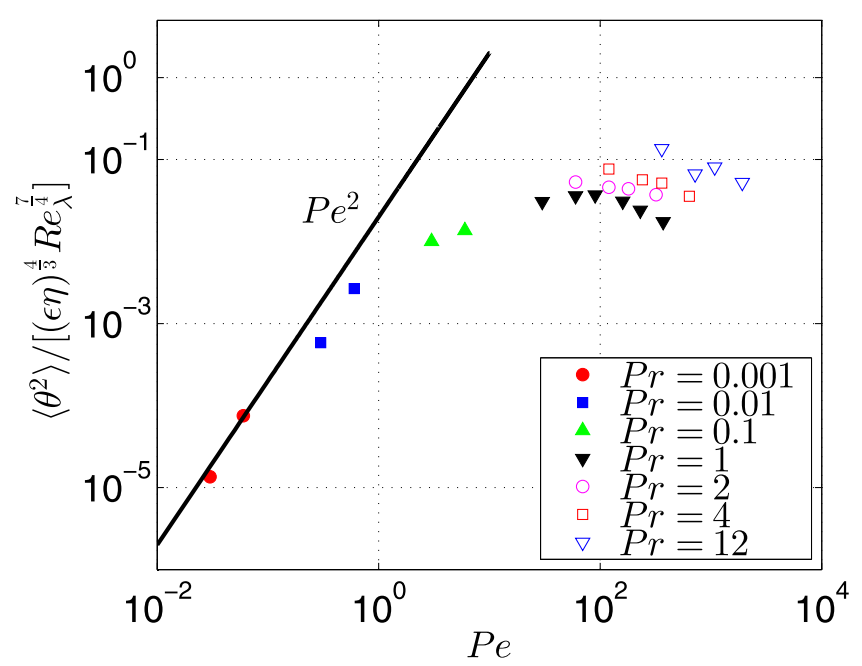

(b)

FIG. 6. (a) Scaling of the normalized dissipation of temperature fluctuations for different Prandtl and Reynolds numbers. (b) Normalized variance of the temperature fluctuations.

values, a constant value should be observed. This is reasonably well confirmed. Similarly, in Fig. 6(b) we test

$$
\left\langle\theta^{2}\right\rangle \frac{c_{p}^{2}}{(\varepsilon \eta)^{4 / 3}} \operatorname{Re}_{\lambda}^{-7 / 4}=F(\mathrm{Pe}),
$$

where now, as predicted, a quadratic dependence is observed at small values, proportional to $\mathrm{Pe}^{2}$, and an approximate plateau is attained at large values. 


\section{CONCLUSION}

We have derived expressions for the statistical description of temperature fluctuations in isotropic turbulence and we have assessed the validity of our predictions using direct numerical simulations. Our simulations suggest that the relevant time scale in the inertial-convective range is a spectrally local time scale of the form $\tau \sim \varepsilon^{-1 / 3} k^{-2 / 3}$ and not the integral time scale as conjectured in Ref. [4].

The spectra of the different quantities collapse in the dissipation range using Kolmogorov variables. For the energy spectrum this collapse is observed for low Reynolds numbers, but for the dissipation-rate fluctuations and the temperature related quantities this collapse is only observed at the highest Reynolds numbers considered in the simulations, for $\operatorname{Re}_{\lambda} \gtrsim 200$. This slow convergence to asymptotic scaling for the temperature variance spectrum is conjectured to be due to the broadband character of the temperature production spectrum and the slow convergence to asymptotic scaling of the dissipation-rate-fluctuation spectrum. Indeed, asymptotically, the temperature-production spectrum should fall off in the inertial-convective range with a power law proportional to $k^{-4 / 3}$. This exponent is in our simulations closer to the exponent -1 , where Corrsin-Obukhov phenomenology breaks down. The Prandtl number does not influence the results significantly for $\operatorname{Pr}>1$, however, in the highly diffusive limit, where $\operatorname{Pr} \ll 1$, the scaling is dominated by a diffusive time scale, leading to a temperature dissipation and variance proportional to $\mathrm{Pe}$ and $\mathrm{Pe}^{2}$, respectively.

To summarize, the present results illustrate that the dissipation-rate spectrum is dominated by intermittent large-scale excitation. The temperature fluctuations are generated by these dissipationrate fluctuations. The spectral distribution of the temperature fluctuations collapses in the dissipation range using Corrsin-Obukhov scaling, but asymptotic scaling is observed only at very high Reynolds numbers. It seems that for the present kind of heat generation, Reynolds numbers of the order of at least $\operatorname{Re}_{\lambda} \approx 10^{3}$ are needed to possibly observe a tendency of the power-law exponent of the temperature spectrum to approach the Corrsin-Obukhov value $-5 / 3$.

\section{ACKNOWLEDGMENTS}

This work was performed using HPC resources from GENCI-IDRIS (Grant No. 020611). The authors thank P. Begou for technical support and data processing. G.B. is grateful for the support from Institut Universitaire de France.

[1] D. De Marinis, S. Chibbaro, M. Meldi, and P. Sagaut, Temperature dynamics in decaying isotropic turbulence with Joule heat production, J. Fluid Mech. 724, 425 (2013).

[2] W. J. T. Bos, The temperature spectrum generated by frictional heating in isotropic turbulence, J. Fluid Mech. 746, 85 (2014).

[3] A. S. Monin and A. M. Yaglom, Statistical Fluid Mechanics II (MIT Press, Cambridge, 1975).

[4] W. J. T. Bos, R. Chahine, and A. V. Pushkarev, On the scaling of temperature fluctuations induced by frictional heating, Phys. Fluids 27, 095105 (2015).

[5] A. S. Gurvich and S. L. Zubkovskii, On experimental estimate of the fluctuations of turbulent energy dissipation, Izv. Akad. Nauk SSSR, Ser. Geofiz. 12, 1856 (1963).

[6] S. Pond and R. W. Stewart, Measurements of the statistical characteristics of small-scale turbulent motions, Izv. Akad. Nauk SSSR, Fiz. Atmos. Okeana 1, 914 (1965).

[7] C. W. Van Atta and W. Y. Chen, Structure functions of turbulence in the atmospheric boundary layer over the ocean, J. Fluid Mech. 44, 145 (1970).

[8] T. Gotoh and R. S. Rogallo, Intermittency and scaling of pressure at small scales in forced isotropic turbulence, J. Fluid Mech. 396, 257 (1999).

[9] Z.-S. She and E. Lévêque, Universal Scaling Laws in Fully Developed Turbulence, Phys. Rev. Lett. 72, 336 (1994). 
[10] H. Chen, J. R. Herring, R. M. Kerr, and R. H. Kraichnan, Non-Gaussian statistics in isotropic turbulence, Phys. Fluids A 1, 1844 (1989).

[11] W. J. T. Bos and R. Rubinstein, On the strength of the nonlinearity in isotropic turbulence, J. Fluid Mech. 733, 158 (2013).

[12] T. Ishihara, Y. Kaneda, M. Yokokawa, K. Itakura, and A. Uno, Spectra of energy dissipation, enstrophy and pressure by high-resolution direct numerical simulations of turbulence in a periodic box, J. Phys. Soc. Jpn. 72, 983 (2003).

[13] R. H. Kraichnan, Lagrangian-history closure approximation for turbulence, Phys. Fluids 8, 575 (1965).

[14] S. Corrsin, On the spectrum of isotropic temperature fluctuations in an isotropic turbulence, J. Appl. Phys. 22, 469 (1951).

[15] A. M. Obukhov, The structure of the temperature field in a turbulent flow, Dokl. Akad. Nauk SSSR 39, 391 (1949).

[16] G. K. Batchelor, I. D. Howells, and A. A. Townsend, Small-scale variation of convected quantities like temperature in turbulent fluid. Part 2, J. Fluid Mech 5, 134 (1959).

[17] K. Alvelius, Random forcing of three-dimensional homogeneous turbulence, Phys. Fluids 11, 1880 (1999).

[18] J.-B. Lagaert, G. Balarac, and G.-H. Cottet, Hybrid spectral-particle method for the turbulent transport of a passive scalar, J. Comput. Phys. 260, 127 (2014).

[19] Y. Kaneda, T. Ishihara, M. Yokokawa, K. Itakura, and A. Uno, Energy dissipation rate and energy spectrum in high resolution direct numerical simulations of turbulence in a periodic box, Phys. Fluids 15, L21 (2003).

[20] Y. Kaneda and T. Ishihara, High-resolution direct numerical simulation of turbulence, J. Turbul. 7, N20 (2006).

[21] T. Watanabe and T. Gotoh, Intermittency in passive scalar turbulence under the uniform mean scalar gradient, Phys. Fluids 18, 058105 (2006). 